\title{
Highly thermally stable heterogeneous catalysts: study of OD and 3D porphyrinic MOFs
}

Received 00th January 20xx, Accepted 00th January 20xx

DOI: $10.1039 / x 0 x x 00000 x$

www.rsc.org/

\author{
E. Amayuelas, ${ }^{a}$ A. Fidalgo-Marijuán, ${ }^{b}$ B. Bazán, ${ }^{*, a, b}$ M. K. Urtiaga, ${ }^{a}$ G. Barandika, ${ }^{b, c}$ and M. I. \\ Arriortua $^{\mathrm{a}, \mathrm{b}}$
}

Heterogeneous catalysts are a great bet for green chemistry in many industrial processes and, in the past decade, promising results have been achieved in order to improve the catalytic activity of Metal Organic Frameworks (MOFs). Accordingly, porphyrins make possible to design new coordination polymers with better properties, taking into account the important functions they develop in nature. In this sense, porphyrin-based MOFs are becoming very relevant in heterogeneous catalysis. Thus, the aim of this work was obtaining metalloporphyrinic MOFs exhibiting catalytic activity. Studying the effect of dimensionality on the MOF properties (including thermal stability and catalytic activity), in this work we study two catalysts with different dimensionalities, 3D $\left[\mathrm{Ni}_{5}\left(\mathrm{H}_{2} \mathrm{TCPP}\right)_{2} \mathrm{O}\left(\mathrm{H}_{2} \mathrm{O}\right)_{4}\right] \cdot \mathrm{nS}(\mathbf{1})$ and $0 \mathrm{D}\left[\mathrm{Cu}\left(\mathrm{H}_{4} \mathrm{TCPP}\right)\right] \cdot 6 \mathrm{DMF}(\mathbf{2})$ (where $\mathrm{H}_{6}$ TCPP is meso-tetra(4-carboxyphenyl)porphyrin, DMF is $\mathrm{N}, \mathrm{N}$-dimethylformamide and $\mathrm{S}$ is the solvent). The structural features of both compounds, combined with their high thermal stability and accessible networks, are responsible for the excellent behaviour as heterogeneous catalysts. It is worth mentioning that significant reduction in reaction time compared to other reported catalysts has been observed. The recyclability of one of the herein studied porphyrin-based MOFs is outstanding. Further structural and thermal characterization has been carried out by means of single crystal X-ray diffraction, IR spectroscopy, thermogravimetry (TG), powder X-ray diffraction (XRD) and transmission electron microscopy (TEM).

\section{Introduction}

Metal organic frameworks (MOFs) have emerged as one of the most interesting materials during this last two decades, due to their chemical and structural features, stability and potential applications in gas sorption, catalysis or drug delivery among others. ${ }^{1-4}$ These porous solids can be constructed from a variety of complexes, as linkers between metal centres, giving rise to a wide variety of crystal structures with different dimensionalities. ${ }^{5,6}$ MOFs of high dimensionalities and high specific surface areas are promising candidates in heterogeneous catalysis, ${ }^{7-9}$ as the big challenge to gain a foothold in reactions where homogeneous catalysts prevail, including hydrocarbons oxidation, alcohols oxidation and Knoevenagel condensation among others. ${ }^{10-14}$

In this context, the selective oxidation of alcohols to aldehydes is a valuable intermediate for the synthesis of many organic compounds, which are widely used in industry, such as pharmaceutical, perfume, plastic, dyestuff and agrochemical. ${ }^{15}$ While considerable progress has been made using noble metal nanoparticles, ${ }^{16,17}$ it would still be desirable to develop catalysts based on less expensive metals and processes. New

\footnotetext{
a. Departamento de Mineralogía y Petrología, Universidad del País Vasco (UPV/EHU), Barrio Sarriena s/n, 48940 Leioa, Spain.

b. BCMaterials, Basque Center for Materials, Applications and Nanostructures, Parque Tecnológico de Zamudio, Ibaizabal Bidea, Edificio 500-Planta 1, 48160 Derio, Spain.

c. Departamento de Química Inorgánica, Universidad del País Vasco (UPV/EHU), Barrio Sarriena $s / n, 48940$ Leioa, Spain.

† Footnotes relating to the title and/or authors should appear here.

Electronic Supplementary Information (ESI) and single-crystal data in crystallographic information files (CIF) format are available. See DOI: $10.1039 / \times 0 x x 00000 x$. X-ray crystallographic information for CCDC No. 1478740
}

approaches following this strategy have been tested for these catalytic reactions, 7,12 making efforts to minimize costs and waste generation. Thus, the great diversity in the design of MOFs and their pore size make these materials to burst into this field with great future perspective for more sustainable industrial processes.

Thus, the use of porphyrins as main ligands is a good strategy to obtain robust frameworks with the desirable porosity. Porphyrins belong to a class of multifunctional biomolecules that play a central role in natural processes in which the transfer of photons, electrons, ions and molecules occurs. ${ }^{18}$ Accordingly, metalloporphyrinic materials can reproduce the natural functions of porphyrins and move them to molecular level structured systems and nanotechnological devices. ${ }^{19,20}$ In this sense, porphyrin-based MOFs are reporting great results, ${ }^{21-23}$ since Suslick et al. reported the first catalytic activity of a porphyrinic coordination polymer with open structure. $^{24}$ In the last years, porphyrinic complexes of iron, ${ }^{25}$ cobalt, ${ }^{26}$ ruthenium, ${ }^{27}$ rhodium, ${ }^{28}$ among others, are known in literature to catalyze chemical reactions like, for example, olefin cyclopropanation and $\mathrm{X}-\mathrm{H}(\mathrm{X}=\mathrm{C}, \mathrm{N}, \mathrm{Si})$ bond insertion with high efficiency and selectivity. For instance, Rh-porphyrin complexes provide cyclopropanation and $\mathrm{C}-\mathrm{H}$ primary insertion products with extraordinary selectivity. ${ }^{28}$ Further, Feand $\mathrm{Ru}$-porphyrins can furnish the $\mathrm{N}-\mathrm{H}$ insertion of ammonia ${ }^{25}$ and peptides containing an $\mathrm{N}$-terminal amine, ${ }^{27}$ respectively. Metalloporphyrinic MOFs combine the versatility of porphyrins, not only with a catalytic function, but also with a structural function which can allow to obtain bigger pores and channels, with the robustness of the structure, making possible in many cases, the recyclability of the material. ${ }^{29}$

At this point, the structure of the material is considered as crucial for the catalytic activity, either because of the metal 
accessibility or the surface area. According to our experience, OD compounds (OD referred to discrete units like monomers or clusters) should not be discarded for catalytic purposes, as long as they have unsaturated accessible metal centers. ${ }^{30,31}$ In fact, our work herein presented consists of the study of two metalloporphyrinic compounds, based on $\mathrm{H}_{6} \mathrm{TCPP}$ (mesotetra(4-carboxyphenyl)porphyrin), in their two possible dimensional extremes, OD and 3D, as heterogeneous catalysts, on alcohols and alkenes oxidation and Knoevenagel condensations. Compound $1,\left[\mathrm{Ni}_{5}\left(\mathrm{H}_{2} \mathrm{TCPP}\right)_{2} \mathrm{O}\left(\mathrm{H}_{2} \mathrm{O}\right)_{4}\right] \cdot \mathrm{nS}$ ( $\mathrm{S}$ is the solvent), is a novel porphyrin-based MOF with big pores and an open 3D structure (with a clear similarity to the reported cobalt compound by Kosal et al., PIZA-1) $)^{32}$. Since this is the first time we report on $\mathbf{1}$, it has been exhaustively characterized by means of single crystal X-ray diffraction, IR spectroscopy, thermogravimetric analysis, powder $\mathrm{X}$-ray diffraction and transmission electron microscopy (TEM). Compound 2, $\left[\mathrm{Cu}\left(\mathrm{H}_{4}\right.\right.$ TCPP) $] \cdot 6 \mathrm{DMF} \quad$ (DMF is $\mathrm{N}, \mathrm{N}$ dimethylformamide), is a OD monomeric compound, and its structure was reported by us elsewhere. ${ }^{33}$

\section{Experimental section}

\section{General}

All solvents and chemicals were used as received from reliable commercial sources. The non-metallated meso-tetra(4carboxyphenyl)porphyrin ( $\left.\mathrm{H}_{6} \mathrm{TCPP}\right)$, nickel(II) nitrate hexahydrate $>98.5 \%$, 1,2,4,5-benzenetetracarboxylic acid $96 \%$ $\left(\mathrm{H}_{4}\right.$ bta) and the solvents $\mathrm{N}, \mathrm{N}$-dimethylformamide 99.8\% (DMF) and acetone $96 \%$ were purchased from Sigma-Aldrich Co.

\section{Synthesis of $\left[\mathrm{Ni}_{5}\left(\mathrm{H}_{2} \mathrm{TCPP}\right)_{2} \mathrm{O}\left(\mathrm{H}_{2} \mathrm{O}\right)_{4}\right] \cdot n \mathrm{nS}(1)$}

meso-tetra(4-carboxyphenyl)porphyrin $(7.9 \mathrm{mg}, 0.01 \mathrm{mmol})$, nickel(II) nitrate hexahydrate $>98.5 \%(29.7 \mathrm{mg}, 0.1 \mathrm{mmol})$ and modulating agent 1,2,4,5-benzenetetracarboxylic acid $96 \%$ (15.7 $\mathrm{mg}, 0.06 \mathrm{mmol}$ ) were dissolved in DMF $(5 \mathrm{~mL})$ in a small capped vial, sonicated to ensure homogeneity and heated to $100{ }^{\circ} \mathrm{C}$ for $72 \mathrm{~h}$, yielding diffraction quality prismatic dark red crystals. This freshly synthesized product was washed with acetone several times before single crystal $X$-ray diffraction experiment. $v_{\max } / \mathrm{cm}^{-1}$ : 3360, $(\mathrm{C}(\mathrm{sp} 2) \mathrm{H}), 2900(\mathrm{OH}), 1670$ $(C=0) \cdot 1600-1390(C=C), 1355(C O), 1310(C=N), 1000(\mathrm{Ni}-$ porphyrin), (Figure S1, Supporting Information).

\section{Single-crystal X-ray diffraction}

Single crystal of compound $\mathbf{1}$ with dimensions given in Table 1 was selected under polarizing microscope and mounted on MicroMounts $^{\mathrm{TM}}$. Single-crystal X-ray diffraction data were collected at $100 \mathrm{~K}$ on an Agilent Technologies SuperNova single source diffractometer with $\mathrm{Cu}-\mathrm{K} \alpha$ radiation $(\lambda=1.54184 \AA$ ). Data frames were processed (unit cell determination, intensity data integration, correction for Lorentz and polarization effects, $^{34}$ and analytical absorption correction) using the CrysAlisPro software package. ${ }^{35}$ The structure of compound 1 was solved in the monoclinic, $\mathrm{C} 2 / \mathrm{m}$ space group, with SHELXS97 program, ${ }^{36}$ which allowed us to obtain the position of metal atoms, as well as nitrogen, oxygen and some of the carbon atoms of the porphyrin. The refinement of the crystal structure was performed by full matrix least-squares based on $F^{2}$, using the SHELXL-97 program ${ }^{36}$ obtaining the remaining carbon atoms. Anisotropic thermal parameters were used for all nonhydrogen atoms (Figure S2, Supporting Information). ${ }^{37}$ All the hydrogen atoms connected to the aromatic rings ( $\mathrm{C}-\mathrm{H} 0.95 \AA \hat{A})$ were fixed geometrically, and were refined using a riding model with common isotropic displacements. Some anisotropic parameters were fixed using EADP instruction. The low crystallinity of the single crystals and the fact that solvent molecules were disordered in the unit cell of $\mathbf{1}$, ended up in the fact that the resulting electron density was found to be non-interpretable. The solvent contribution to the structure factors was taken into account by back-Fourier transformation of all the densities found in the disordered area using the SQUEEZE tool in PLATON. ${ }^{38}$ The calculated density does not take into account the solvent, causing disagreement between calculated and experimental density. Some hydrogen atoms of the carboxylic groups were not considered due to the lack of density in the residual density map; however they are included in the formula. Atomic coordinates, anisotropic thermal parameters and bond distances and angles are given in Tables S1-S3 in Supporting Information.

\section{Physicochemical characterization techniques}

The IR spectra were collected on a JASCO FT/IR-6100 spectrometer at room temperature at the range of $4000-400$ $\mathrm{cm}^{-1}$, in $\mathrm{KBr}$ pellets ( $1 \%$ of the sample). Thermogravimetric analyses were carried out using a NETZSCH STA 449F3 thermobalance, where a crucible containing approximately 10 $\mathrm{mg}$ of sample was heated at $5{ }^{\circ} \mathrm{C} \mathrm{min}^{-1}$ in the temperature range $30-600^{\circ} \mathrm{C}$. BET 
Table 1. Crystallographic data of compound 1.

\begin{tabular}{|c|c|}
\hline Compound & 1 \\
\hline structural formula & {$\left[\mathrm{Ni}_{5}\left(\mathrm{H}_{2} \mathrm{TCPP}\right)_{2} \mathrm{O}\left(\mathrm{H}_{2} \mathrm{O}\right)_{4}\right] \cdot \mathrm{nS}$} \\
\hline empirical formula & $\mathrm{C}_{96} \mathrm{H}_{52} \mathrm{~N}_{8} \mathrm{Ni}_{5} \mathrm{O}_{21}$ \\
\hline $\mathrm{F}_{\mathrm{w}}, \mathrm{g} \mathrm{mol}^{-1}$ & 1942.87 \\
\hline cryst. system & Monoclinic \\
\hline space group & $\mathrm{C} 2 / \mathrm{m}$ \\
\hline$a, \AA$ & $17.640(4)$ \\
\hline$b, \AA$ & $33.509(2)$ \\
\hline$c, \AA$ & $16.6141(17)$ \\
\hline$\beta$, deg & $103.793(14)$ \\
\hline$V, \AA^{3}$ & $9538(3)$ \\
\hline Z & 2 \\
\hline$\rho_{\text {obs }}, \rho_{\text {cal }}, \mathrm{g} \cdot \mathrm{cm}^{-3}$ & $1.546(5), 0.677^{*}$ \\
\hline Crystal size, $\mathrm{mm}$ & $0.12 \times 0.11 \times 0.02$ \\
\hline$\mu, \mathrm{mm}^{-1}$ & 0.844 \\
\hline absorption correction & Analytical \\
\hline radiation, $\lambda, \AA$ & 1.54184 \\
\hline temperature, $\mathrm{K}$ & $100.0(2)$ \\
\hline reflns. collected, unique & $7925,3407\left(R_{\text {int }}=0.147\right)$ \\
\hline final $R$ indices $[I>2 \sigma(I)]$ & $\mathrm{R} 1=0.0991, w R 2=0.2522$ \\
\hline $\mathrm{R}$ indices (all data) & $R 1=0.1627, w R 2=0.2919$ \\
\hline GOF on $\mathrm{F}^{2}$ & 0.952 \\
\hline parameters / restraints & $264 / 0$ \\
\hline
\end{tabular}

*Differences between observed and calculated density due to the SQUEEZE tool in the structure refinement.

\section{Catalytic Tests}

The oxidation reactions of benzyl alcohol, 1-phenylethanol, 4chlorobenzyl alcohol, 4-methylbenzyl alcohol, 1-hexanol, 1octanol (Scheme 1a) and $\beta$-methylstirene (Scheme 1b) were carried out at $100{ }^{\circ} \mathrm{C}$ using acetonitrile as solvent. The catalyst/substrate molar ratio (based on metal) used for all the reactions is $5 / 100$. Powdered crystals of catalysts were firstly dried under vacuum at $200{ }^{\circ} \mathrm{C}$ for compound 1 and at $150{ }^{\circ} \mathrm{C}$ for compound $\mathbf{2}$ to remove solvent and water adsorbed on the surface. Before the reactions, approximately $5 \mathrm{mg}$ of dried catalyst were activated by stirring it with the oxidizing agent, tert-butyl hydroperoxide (TBHP), in $2 \mathrm{~mL}$ of acetonitrile, for 30 $\min$ at $100{ }^{\circ} \mathrm{C}$. After this activation stage, the catalyst was separated from the liquid media by centrifugation. The reactor was then charged with the activated catalyst, and the corresponding alcohol in $2 \mathrm{~mL}$ of solvent. The mixture was heated up to $100{ }^{\circ} \mathrm{C}$ and then the oxidizing agent was added dropwise (1.5 eq. of TBHP). Knoevenagel condensation reactions of benzaldehyde, $p$-tolualdehyde, $p$ fluorubenzaldehyde and $p$-chlorobenzaldehyde (Scheme 1c) were carried out at $100{ }^{\circ} \mathrm{C}$ using toluene as solvent. The catalyst/substrate molar ratio (based on metal) used for all the reactions is 5/100. Powdered crystals of catalysts $\mathbf{1}$ and $\mathbf{2}$ were firstly dried at 200 and $150{ }^{\circ} \mathrm{C}$, respectively, under vacuum to remove solvent and water adsorbed at the surface. The reactor was charged with the catalyst $(5 \mathrm{mg})$, malononitrile (4.3 $\mathrm{mg}$ for catalyst 1 and $6.1 \mathrm{mg}$ for 2 ), dodecane $(2.0 \mu \mathrm{L}$ ) and the corresponding substrate in $2 \mathrm{~mL}$ of solvent, then the mixture was heated up to $100^{\circ} \mathrm{C}$.

Solutions were analysed on a 7890A Agilent gas chromatograph coupled to a $5975 \mathrm{C}$ inert XL Agilent mass spectrometer (Agilent Technologies, Avondale, PA, USA) with a Combi PAL autosampler (CTC Analytics, Zwingen, Switzerland). An amount of $2 \mu \mathrm{L}$ of the sample was injected in the split mode $(1: 20)$ at $270{ }^{\circ} \mathrm{C}$ into a $30 \mathrm{~m} \times 0.25 \mathrm{~mm} \times 0.25 \mu \mathrm{m}$ DB-WAX capillary column. The following temperature programme was used: $80^{\circ} \mathrm{C}$ for $2 \mathrm{~min}$, temperature increase from $12^{\circ} \mathrm{Cmin}^{-1}$ to $210{ }^{\circ} \mathrm{C}$ where it was finally held for $5 \mathrm{~min}$. The carrier gas was helium $(\mathrm{N}-50)$ at a constant flux of $1.4 \mathrm{~mL} \mathrm{~min}{ }^{-1}$. The mass spectrometer was operated in the electron impact ionisation mode and the energy of the electrons was kept at $70 \mathrm{eV}$. The interface was kept at $300{ }^{\circ} \mathrm{C}$ and the ionisation source and the quadrupole at 230 and $150{ }^{\circ} \mathrm{C}$, respectively. Measurements in the GC-MS were performed in the SCAN mode. After the reaction, the catalysts were filtered, dried and characterised by IR spectroscopy and Transmission Electron Microscopy (TEM).

The calculations of turnover frequencies (TOF: mol substrate converted per mol catalyst per hour) were calculated in the initial stages of the reaction, when the reaction rates are higher, as usual.

High Resolution TEM measurements were carried out on a FEI Titan Cubed G2 60-300 microscope, equipped with a Schottky $\mathrm{X}$-FEG field emission electron gun, monochromator and CEOS $\mathrm{GmbH}$ spherical aberration (Cs) corrector on the image side. The microscope was operated at $80 \mathrm{kV}$ to minimize the knockon damage. The third-order spherical aberration (Cs) was tuned to $6 \mu \mathrm{m}$. Images were recorded on a CCD camera (2kx2k, Gatan UltraScan 1000), using exposition times of 1s per image. The samples for the TEM were prepared by dispersion into ethanol solvent and keeping the suspension in an ultrasonic bath for $15 \mathrm{~min}$, after a drop of suspension was spread onto a TEM copper grid (300 Mesh) covered by a holey carbon film followed by drying under vacuum.

\section{Results and Discussion}

\section{Crystal structures}

Compound 1, with formula $\left[\mathrm{Ni}_{5}\left(\mathrm{H}_{2} \mathrm{TCPP}\right)_{2} \mathrm{O}\left(\mathrm{H}_{2} \mathrm{O}\right)_{4}\right] \cdot \mathrm{nS}$ arrays in a 3D framework. This compound and PIZA-1 by Kosal et al., ${ }^{32}$ show similar structural features but PIZA-1 is Co"-based.

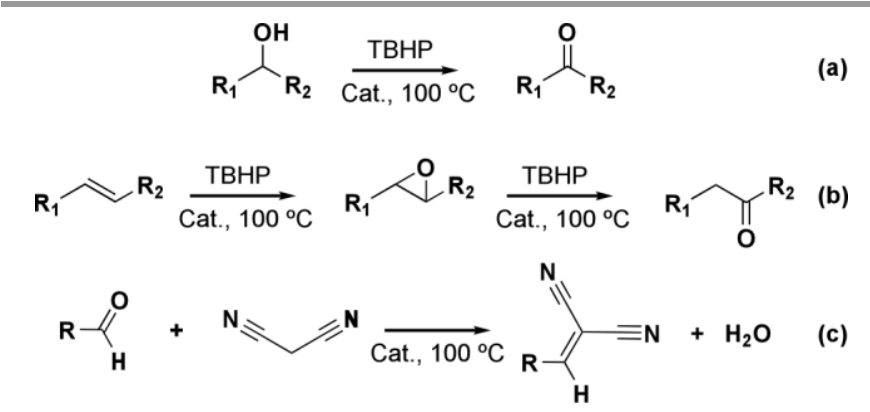

Scheme 1. Catalytic tests reactions for (a) oxidation of alcohols, (b) oxidation of alkenes and (c) Knoevenagel condensation. 
Crystal structure of $\mathbf{1}$ consists of $\mathrm{Ni}^{\mathrm{II}}$ metalloporphyrin layers over yz plane linked by trinucluear (Figure 1 ) clusters of $\mathrm{Ni}^{\prime \prime}$. There are three crystallographically independent $\mathrm{Ni}$ atoms. One of them is located in the centre of the porphyrin (Ni1), in a square planar coordination environment, linked to four $\mathrm{N}$ atoms. These metalloporphyrinic units are linked by centrosymmetric trinuclear entities formed by octahedrally coordinated $\mathrm{Ni}$ atoms ( $\mathrm{Ni} 2$ and $\mathrm{Ni3}$ ). This arrangement gives rise to a $3 D$ structure, showing connected channels, with the following dimensions: $13.3 \AA$ x $13.3 \AA$ (along [100]) and $23.7 \AA$ $x 8.8 \AA$ (along [112]), where the dimensions are atom-to-atom distances. Those voids are occupied by DMF and water solvent molecules. Unfortunately, we have not been able to localize them on the electronic density map during crystal structure refinement.

Trinuclear centrosymmetric clusters (Figure 2), formed by two $\mathrm{Ni}^{\prime \prime}$ atoms ( $\mathrm{Ni2}$ and $\mathrm{N} 3$ ), link 8 porphyrinic units building a robust framework. Each in-cluster metal atom is coordinated to six oxygen atoms; four of them belonging to carboxylate groups from lateral substituent of $\mathrm{H}_{2}$ TCPP and, in the case of $\mathrm{Ni} 2$ (the central atom of the cluster, lying on a special position), two oxygen atoms acting as oxo bridges. $\mathrm{Ni3}$ is coordinated to an equatorial water molecule and an oxygen atom as oxo bridge which completes the octahedral

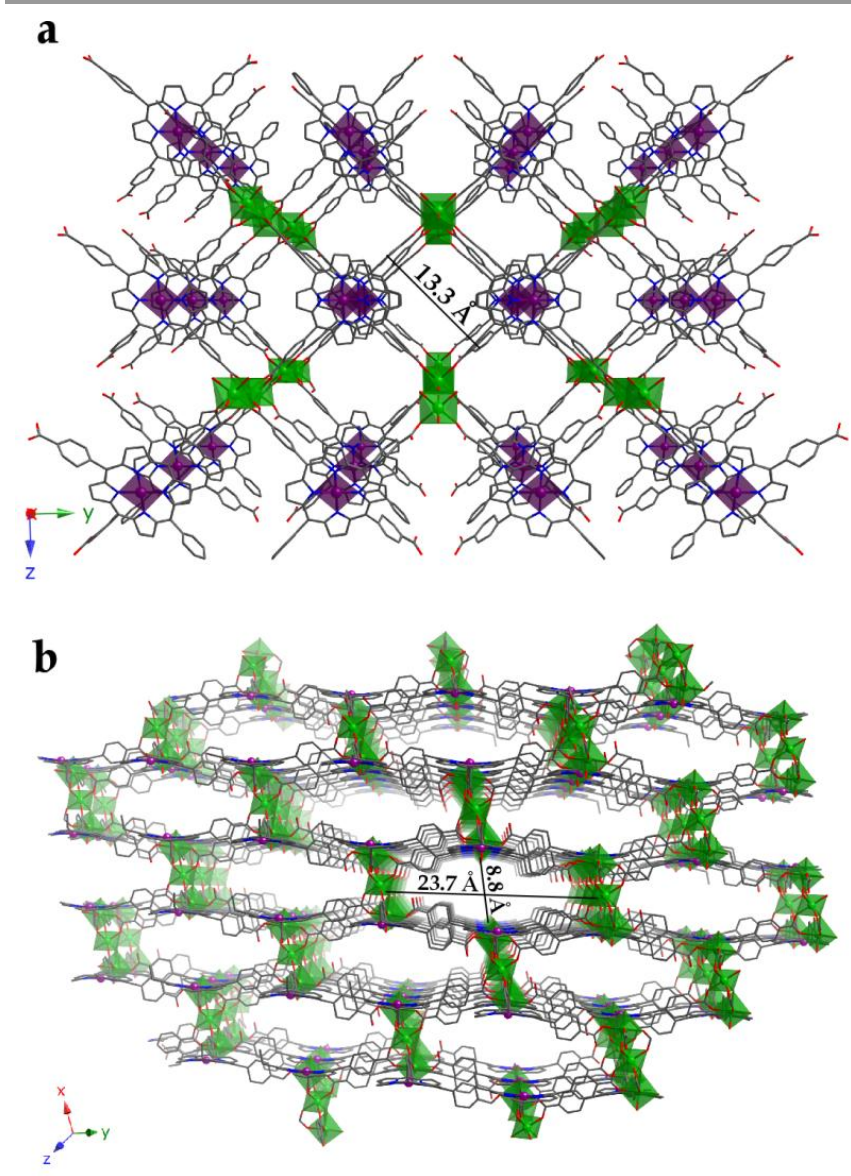

Figure 1. a) [100] and b) [112] views of the 3D framework of compound 1. (Nipor: purple, Nitri: green, C: grey, N: dark blue, O: red). $\mathrm{H}$ atoms have been omitted for clarity.

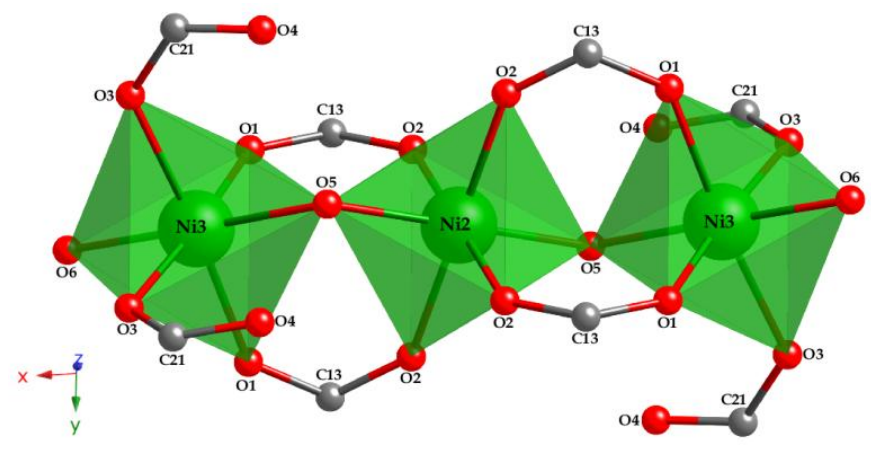

Figure 2. View of the trinuclear cluster of $\mathrm{Ni}$ for compound $\mathbf{1}$

coordination environment. Therefore, $\mathrm{Ni} 2$ and $\mathrm{Ni} 3$ are connected by means of two linkers: by carboxylate groups (Ni3-O1-C13-O2-Ni2), as the long route and by oxo bridges (Ni3-O5-Ni2) as the short route, as shown in Figure 2.

Distortion of coordination polyhedra has been evaluated according to Avnir et al.39 method, based on the continuous symmetry measures (CSM), by means of SHAPE program, 40 and the results can be seen in Table 2. The projection of the as-calculated values on the distortion diagram ${ }^{41}$ can be seen in Figure S3, Supporting Information. Values for Ni1 show that the square-planar coordination environment is distorted from the ideal square, due to the stress present between trinuclear clusters and metalloporphyrins which ruffles the porphyrin macrocycle. In the case of coordination polyhedra for $\mathrm{Ni} 2$ and $\mathrm{Ni3}$, the results indicate that octahedra are nearly ideal. However, values for $\mathrm{Ni} 3$ show a slight Jahn-Teller distortion. Bond distances and angles are reported in Table S4 in Supporting Information. Finally, a BET surface area of 320.33 $\mathrm{m}^{2} / \mathrm{g}$ was calculated for 1 (Figure S4, Supporting Information). As said before, crystal structure of compound $\mathbf{2}$ was reported by us elsewhere ${ }^{33}$. This compound, $\left[\mathrm{Cu}\left(\mathrm{H}_{4} \mathrm{TCPP}\right)\right] \cdot 6 \mathrm{DMF}$, shows $\mathrm{CuH}_{4}$ TCPP monomeric units. The copper atom is in a fourcoordinated square planar linked to four $\mathrm{N}$ atoms in the centre of the tetrapyrrolic unit. These coordination entities crystallise as shown in Figure 3, where each porphyrinic unit is surrounded by another six, producing an $\mathrm{H}$-bonded 2D layer on the $y z$ plane. The robust intralayer $\mathrm{H}$-bonding system involving the DMF solvent molecules located between porphyrins molecules maintains the stability of the supramolecular layers. Those are stacked along the [100] direction sustained by hydrogen bonds among the monomers and DMF molecules of each layer.

Table 2. Distortion values calculated for the hexacoordinated spheres of $\mathrm{Ni} 2$ and $\mathrm{Ni} 3$ in compound $\mathbf{1}$ (calculated by means of SHAPE software).

\begin{tabular}{cccc}
\hline & & & \\
\hline Compound 1 & Tetracordinate & $\mathrm{S}\left(D_{4 \mathrm{~h}}\right)$ & $\mathrm{S}\left(T_{\mathrm{d}}\right)$ \\
\hline & $\mathrm{Ni} 1$ & 0.006 & 33.56 \\
\hline \multirow{2}{*}{ Compound 1 } & Hexacoordinate & $\mathrm{S}\left(O_{\mathrm{h}}\right)$ & $\mathrm{S}\left(D_{3 \mathrm{~h}}\right)$ \\
\cline { 2 - 4 } & $\mathrm{Ni2}$ & 0.037 & 16.56 \\
\hline
\end{tabular}




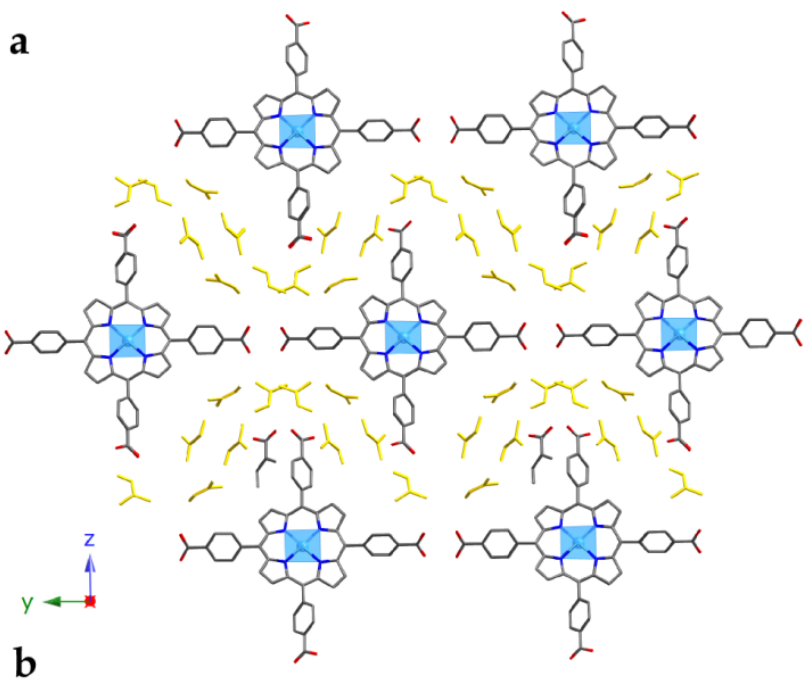

b

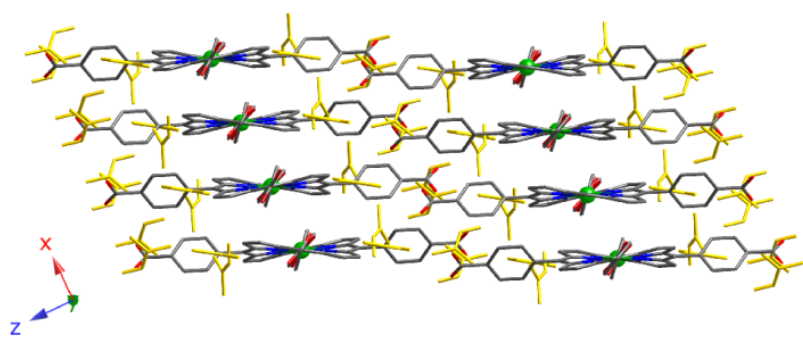

Figure 3. (a) View of the $\mathrm{H}$-bonded 2D supramolecular layer and (b) packing fo compound 2. (Cu: turquoise, $\mathrm{C}$ : grey, $\mathrm{N}$ : dark blue, $\mathrm{O}$ : red, crystallization $\mathrm{DM}$
molecules: yellow). $\mathrm{H}$ atoms have been omitted for clarity.

\section{Thermal analysis}

In order to analyze the thermal behaviour and stability of compound 1 , thermogravimetric and IR measurements were performed. IR spectra were recorded on a heated, powdered sample taken immediately after synthesis.

\section{Thermogravimetric analysis.}

The thermogravimetric decomposition curve shows a twostage mass loss (Figure 4). First step occurs between $25-260{ }^{\circ} \mathrm{C}$ with a total of $17.5 \%$ weight loss (attributed to the removal of DMF and water solvent molecules). However, the 3D structure remains stable as shown by IR spectroscopy (Figure S5, Supporting Information). The second significant process, from $320{ }^{\circ} \mathrm{C}$ to $420{ }^{\circ} \mathrm{C}$ ( $65 \%$ weight loss), has been attributed to the calcinations of the TCPP units. The residue (17.5\%) has been identified by X-ray powder diffraction as NiO [S. G. Fm-3m, a = $4.17 \AA]^{42}$. Therefore, thermal stability of compound 1 is remarkably high.

\section{Catalytic properties}

As the aim of this work is to study the catalytic activity of two porphyrin-based MOFs with different dimensionalities, the 3D compound $1\left(\left[\mathrm{Ni}_{5}\left(\mathrm{H}_{2} \mathrm{TCPP}\right)_{2} \mathrm{O}\left(\mathrm{H}_{2} \mathrm{O}\right)_{4}\right] \cdot \mathrm{nS}\right)$ and the $\mathrm{OD}$ compound 2 ([Cu( $\left.\left.\left.\mathrm{H}_{4} \mathrm{TCPP}\right)\right] \cdot 6 \mathrm{DMF}\right)$ have been tested towards the oxidation of alcohols and Knoevenagel condensation. Those two solid networks exhibit some features that make us think of its potential as a catalyst. Firstly, the metal atoms in the center of the tetrapyrrolic units are unsaturated.

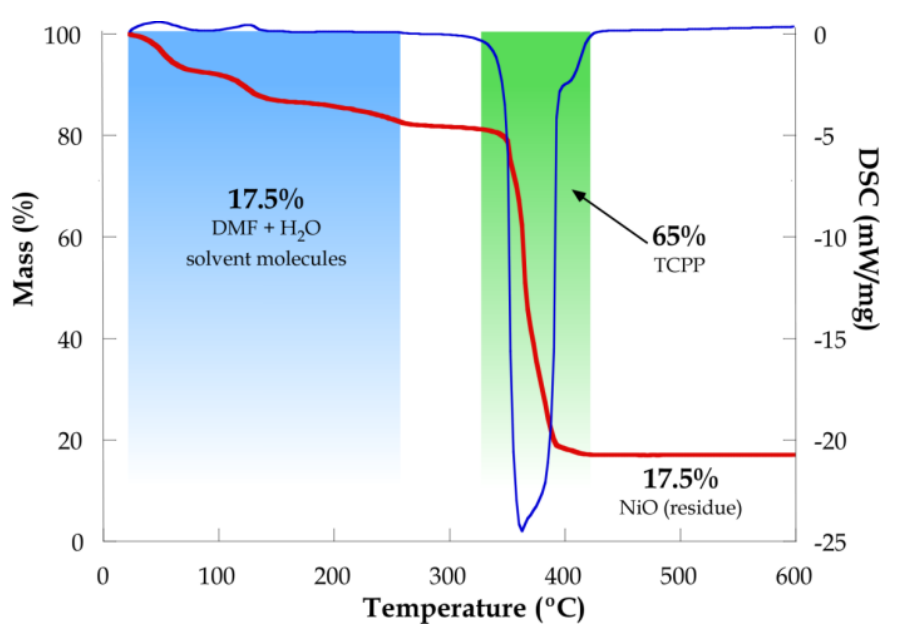

Figure 4. TG (red) and DSC (blue) curves for compound 1.

In addition, the networks are significantly accessible, with mobile DMF or water solvent molecules located in the cavities, easy to remove in an activation stage, which can be carried out due to the high thermal stability of both compounds.

\section{Oxidation of alcohols and alkenes}

The reactions conditions were previously set using benzyl alcohol as model substrate. Based on our previous experience, the reactions have been carried out with TBHP as oxidizing agent in acetonitrile. Using a $5 \%$ of catalyst, 2 eq. of TBHP in 2 $\mathrm{mL}$ of solvent at $100{ }^{\circ} \mathrm{C}$ a total conversion of $42 \%$ for 1 and $54 \%$ for 2 has been achieved after 24 hour of reaction. The substrate scope has been studied with various alcohols and one alkene: 1-phenylethanol, 4-chlorobenzyl alcohol, 4methylbenzyl alcohol, 1-hexanol, 1-octanol and the alkene $\beta$ methylstirene (Table 3 ). Figure S6 in Supporting Information shows the kinetic profiles of the oxidation reactions for $\mathbf{1}$ and 2, respectively. As shown in table 3 , for both compounds, the conversion rates for aromatic alcohols are higher than for the linear ones, probably due to the fact that these substrates present more steric hindrance to access de active centres. For the oxidation of the $\beta$-methylstirene, the reaction evolves very rapid, achieving the total oxidation of the substrate in only $4 \mathrm{~h}$. In addition, when the reaction reaches $90 \%$ of the total conversion using catalyst $\mathbf{1}$, the intermediate product (2methyl-3-phenyl-oxyrane), with an epoxy group, is formed at the same time as the formation of an overoxydized product begins, benzyl methyl ketone, reaching a $68 \%$ of epoxy product and $32 \%$ of the ketone product up to $24 \mathrm{~h}$. This selectivity could allow to control the reaction and take advantage of intermediate product.

In any case, it is clearly shown that the TOF values for compound $\mathbf{2}$ are much higher reaching high conversion values on the initial stage of the reaction. A comparison of those results with similar porphyrinic catalysts found in literature indicates a significant increase of the conversion values using $5 \%$ of catalyst and in similar reaction conditions. ${ }^{43}$ Moreover, comparing the results with classic $\mathrm{Rh}, \mathrm{Ru}$ and $\mathrm{Ce}$ based catalysts, the conversion rates are slightly higher and much shorter reactions for compound $\mathbf{1}$ and $\mathbf{2}{ }^{44-46}$ 
One of the disadvantages of the heterogeneous catalysts is the difficulty to study the reaction, and often the involved mechanisms are unknown. Even so, in the proposed mechanism for the alcohols and alkenes oxidation, the initial stage could consist of the activation of the TBHP by coordination to the unsaturated $\mathrm{Ni}$ and $\mathrm{Cu}$ centers in order to obtain the corresponding peroxo species. After coordination, $t$ - butoxyl radicals would be generated, extracting a hydrogen atom from the substrate leading to the corresponding aldehyde or ketone. The accessible centers are in both cases the metal ions coordinated to porphyrins.

Table 3. Selective oxidation of several alcohols and alkenes over $\mathbf{1}$ and $\mathbf{2}$ catalysts.

\begin{tabular}{|c|c|c|c|c|c|c|c|c|c|}
\hline \multirow[b]{2}{*}{ Substrate } & \multirow[b]{2}{*}{ Oxidant } & \multicolumn{4}{|c|}{ Compound 1} & \multicolumn{4}{|c|}{ Compound $\mathbf{2}$} \\
\hline & & $\operatorname{TOF}^{a}\left(h^{-1}\right)$ & $C_{T}^{b}(\%)$ & Selectivity (\%) & Time (h) & $\operatorname{TOF}^{a}\left(h^{-1}\right)$ & $\mathrm{C}_{\mathrm{T}}^{\mathrm{b}}(\%)$ & Selectivity (\%) & Time (h) \\
\hline Benzyl alcohol & TBHP & 14.2 & 42 & 100 & 24 & 64.8 & 54 & 100 & 24 \\
\hline 1-phenylethanol & TBHP & 46 & 100 & 100 & 24 & 135.6 & 100 & 100 & 24 \\
\hline 4-chlorobenzyl alcohol & TBHP & 65.1 & 80 & 100 & 24 & 156.3 & 80 & 100 & 24 \\
\hline 4-methylbenzyl alcohol & TBHP & 21 & 98 & 100 & 24 & 129 & 71 & 100 & 24 \\
\hline 1-hexanol & TBHP & 13.63 & 56 & 100 & 24 & 3.85 & 16 & 100 & 24 \\
\hline 1-octanol & TBHP & 4.7 & 35 & 100 & 24 & 6.66 & 32 & 100 & 24 \\
\hline$\beta$-methylstirene & TBHP & 51.78 & 100 & 68 & 4 & 156.6 & 100 & 100 & 4 \\
\hline
\end{tabular}

${ }^{\mathrm{a}} \mathrm{TOF}$ : mol subst. conv. per mol cat. h. ${ }^{\mathrm{b}} \mathrm{C}_{\mathrm{T}}$ : Total conversion.

Knoevenagel condensation.

Compounds $\mathbf{1}$ and $\mathbf{2}$ have also been tested to catalyze the Knoevenagel condensation reaction between benzaldehyde and derivatives and malononitrile $\left(\mathrm{pK}_{\mathrm{a}}=11.1\right)$. As above, the reaction conditions were set using benzaldehyde as substrate, $5 \%$ of catalyst, 1.2 eq. of malononitrile, 0.5 eq. of dodecane (internal standard) in $2 \mathrm{~mL}$ of toluene at $100{ }^{\circ} \mathrm{C}$, reaching a total conversion of $36 \%$ after 24 hours of reaction (Table 4 ). The substrate scope was then studied with $p$-tolualdehyde, $p$ fluorobenzaldehyde and p-chlorobenzaldehyde. Figure S7 in Supporting Information shows the kinetic profiles of the
Knoevenagel condensation reactions and, as observed, the reactions evolve slowly with both catalysts. A possible reason could be the proximity of the active centers allowing the cyanide groups of malononitrile to interact with the metal catalytic centres. In the case of compound $\mathbf{1}$, the intermetallic distance between porphyrinic centers is higher producing a lower conversion. Related to compound 2, shortest intermetallic distance between Cu centers is $3.8 \AA$ along [100] (Figure S8, Supporting Information). This space is not accessible enough to reach higher conversions.

Table 4. Knoevenagel condensations over $\mathbf{1}$ and $\mathbf{2}$ catalysts.

\begin{tabular}{|c|c|c|c|c|c|c|c|c|}
\hline \multirow[b]{2}{*}{ Substrate } & \multicolumn{4}{|c|}{ Compound 1} & \multicolumn{4}{|c|}{ Compound 2} \\
\hline & $\operatorname{TOF}^{\mathrm{a}}\left(\mathrm{h}^{-1}\right)$ & $\mathrm{C}_{\mathrm{T}}^{\mathrm{b}}(\%)$ & Selectivity (\%) & Time (h) & $\operatorname{TOF}^{a}\left(h^{-1}\right)$ & $\mathrm{C}_{\mathrm{T}}^{\mathrm{b}}(\%)$ & Selectivity (\%) & Time (h) \\
\hline Benzaldehyde & 7.2 & 36 & 100 & 24 & 6.9 & 36 & 100 & 24 \\
\hline$p$-tolualdehyde & 12 & 50 & 100 & 24 & 7.8 & 35 & 100 & 24 \\
\hline$p$-fluorobenzaldehyde & 3.75 & 19 & 100 & 24 & 6.6 & 26 & 100 & 24 \\
\hline$p$-chlorobenzaldehyde & 1.2 & 3 & 100 & 24 & 40.8 & 17 & 100 & 24 \\
\hline
\end{tabular}

${ }^{\mathrm{a}} \mathrm{TOF}$ : mol subst. conv. per mol cat. $\mathrm{h} .{ }^{\mathrm{b}} \mathrm{C}_{\mathrm{T}}$ : Total conversion.

\section{Heterogeneity and recyclability tests}

The heterogeneity nature of the catalysts $\mathbf{1}$ and $\mathbf{2}$ towards the oxidation of alcohols and Knoevenagel condensation was tested using benzyl alcohol and benzaldehyde, respectively. For rigorous proof of heterogeneity, the tests ${ }^{47}$ were carried out by filtering the catalyst from the reaction mixture at $100{ }^{\circ} \mathrm{C}$ after $2 \mathrm{~h}$, when a conversion of $18 \%$ and $37 \%$ had been reached for the oxidation and $12 \%$ and $10 \%$ for the condensation for $\mathbf{1}$ and $\mathbf{2}$, respectively. The filtrate was allowed to react for up to $6 \mathrm{~h}$. The reaction mixture and the filtrate were analyzed then by GC-MS. No significant changes in the conversion rates were found for the filtrate (Figures S9 and S10, Supporting Information), meaning that the active species do not leach and the observed catalysis is truly heterogeneous in nature.

In order to analyze the reusability of the catalysts, the recyclability of $\mathbf{1}$ and $\mathbf{2}$ was also tested for both reactions. The catalysts were recovered after the reaction by centrifugation and washed several times with acetonitrile or toluene, then 
dried at $100{ }^{\circ} \mathrm{C}$ and reused. As shown in Figure 5 a, catalyst 1 maintains the catalytic activity for Knoevenagel condensations after five cycles, while for oxidations of alcohols the conversion rates slightly increases, probably due to the catalytically active species formed in the presence of the oxidant increases after the first run. For $\mathbf{2}$, the conversion rates of oxidations remain stable during the five cycles, while for the condensation reactions a broad decrease is observed meaning that the $\mathrm{Cu}$ active centers gradually lose the catalytic efficiency or they become less accessible to fresh substrates (Figure 5b).

After the catalytic reactions, the solid of $\mathbf{1}$ and $\mathbf{2}$ catalysts was recovered by centrifugation, washed with acetonitrile or toluene and then characterized by IR spectroscopy and powder X-ray diffraction. The IR spectra of the recovered catalyst for the all reactions show that the structural units remain stable; in fact, the solid shows the same characteristic vibration modes exhibited by the original compound. As shown in Figure S11 in Supporting Information, the characteristic vibrations of the porphyrin macrocycle are present. XRD patterns for samples of $\mathbf{1}$ after catalysis (Figure S12, Supporting Information) show no displacements in characteristic peaks compared with XRD pattern of freshly synthesized sample, confirming the stability of 1 . However, comparison of XRD patterns for samples of compound $\mathbf{2}$ before and after catalysis (Figure S12, Supporting Information), exhibits a remarkable displacement, suggesting a structural change. Nevestheless, due to the low crystallinity of compounds $\mathbf{1}$ and 2, these results should not be decisive to evaluate the heterogeneity of these catalysts. Thus, both the fresh catalyst and the recovered solid after the reaction were studied by TEM, as shown below. a

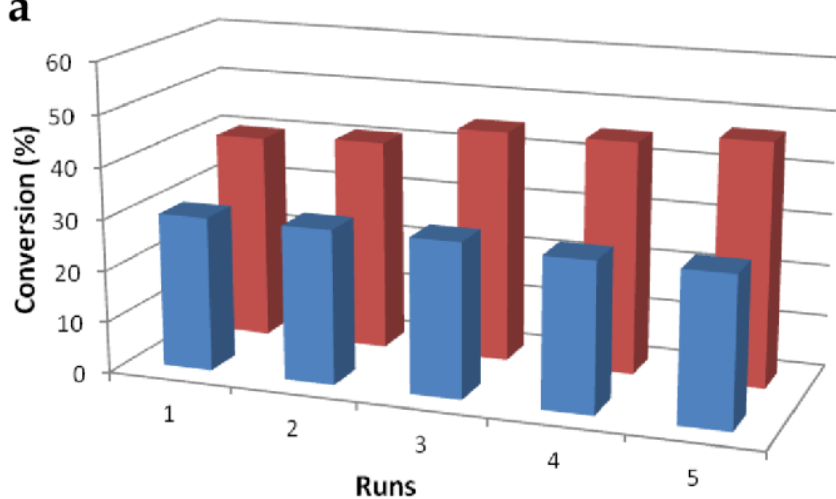

b Knoevenagel cond. $\quad$ Oxidations

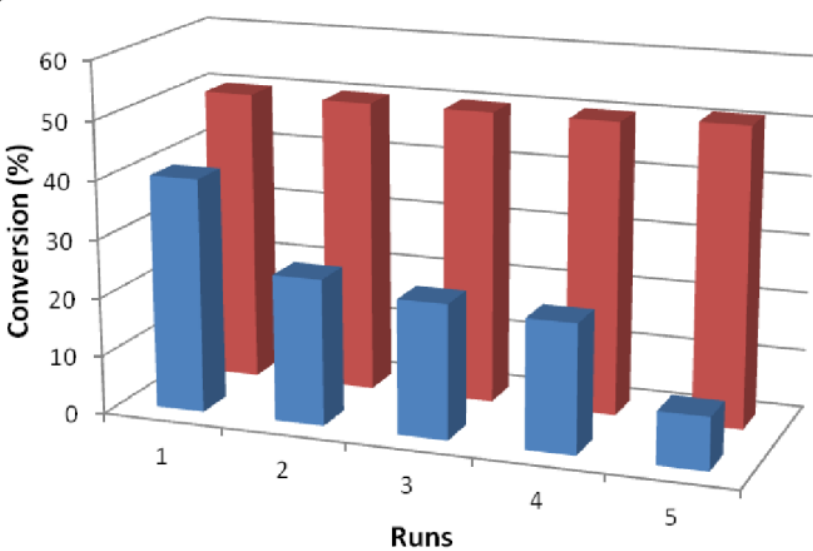

Figure 5. Recycling experiments for (a) compound 1 and (b) compound 2.

\section{Transmission Electron Microscopy (TEM).}

In order to support heterogeneity and recyclability properties for compounds $\mathbf{1}$ and 2, TEM analysis of the catalysts were carried out before and after the catalytic reactions. The results confirm that compounds $\mathbf{1}$ and $\mathbf{2}$ keep structural integrity after the catalytic reactions. Whereas pristine and post-catalysis particles of compound $\mathbf{1}$ maintain the morphology, particles of compound $\mathbf{2}$ are degraded losing slightly their elongated morphology as shown in Figure S13, in Supporting Information. High Resolution TEM images show crystalline nature for pristine compounds and for samples recovered after catalytic tests. Thus, the lattice spacing for both compounds has been measured. Lattice spacing values of $13.69 \AA$ and $14.08 \AA$ have been observed for pristine compound 1 and after catalytic sample respectively, indicating the occurrence of no significant changes in structural parameters. The lattice spacing has been assigned to the (200) set of planes, so it is worth mentioning that the spacing values ( $13.69 \AA$ and $14.08 \AA$ ) are remarkably close to the value of $13.3 \AA$ observed in Figure 1 a. For compound 2, measured spacing values before and after catalytic tests are $14.1 \AA$ and $17.24 \AA$, respectively (Figure 6). Therefore, spacing values for compound 1 confirm the recyclable nature of the catalyst while this has not been possible for compound $\mathbf{2}$. In spite of that, both compounds keep their structural integrity as confirmed by IR spectroscopy and XRD (Figures S11 and S12, Supporting Information). 

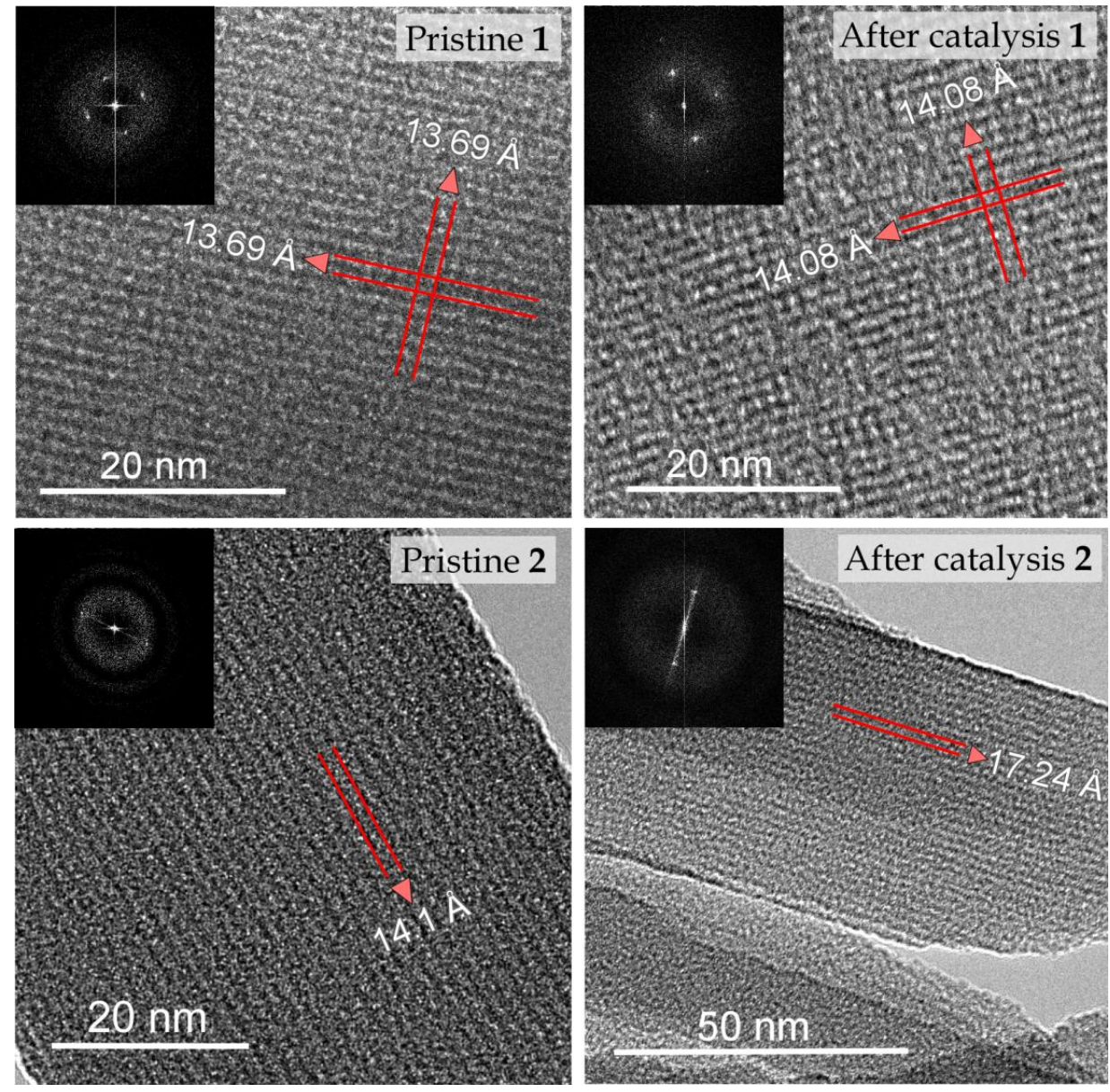

Figure 6. HRTEM images for pristine compounds $\mathbf{1}$ and $\mathbf{2}$ and the recovered residues after the catalytic reactions for both compounds. Lattice spacing is marked with red lines and the upper left image corresponds to the Fourier Transform (FFT).

\section{Conclusions}

A new, robust 3D porphyrin-based MOF (compound 1) has been synthesized showing accessible microporosity and high thermal stability. Due to these outstanding properties, this compound and compound $\mathbf{2}$ have been tested as catalysts in heterogeneous conditions. Catalytic tests show that both compounds are very effective as catalysts; concretely towards oxidation reactions. In the case of compound 2, TOF values for aromatic alcohols and alkenes oxidation are significantly high. These results contribute to reinforce the idea that the accessibility to unsaturated metal centres can be as significant as dimensionality and surface area for the catalytic activity, which is in accordance with our previous results. Compound 1 has been confirmed to be selective towards the oxidation of one studied substrates. An additional advantage of $\mathbf{1}$ lies on its recyclability, related to its robust structural framework as a crucial feature for heterogeneous catalysts.

\section{Acknowledgements}

This work has been financially supported by the "Ministerio de Economia, Industria y Competitividad" (MAT2016-76739-R), and the "Gobierno Vasco" (Basque University System
Research Groups, IT-630-13) which are gratefully acknowledge. The authors acknowledge the support received by the European Regional Development Fund (ERDF). The technical and human support provided by SGIker (UPV/EHU) is gratefully acknowledged. E. Amayuelas thanks the University of the Basque Country for his postdoctoral fellowship.

\section{Notes and References}

1. J. Liu, P. K. Thallapally, B. P. McGrail, D. R. Brown and J. Liu, Chem. Soc. Rev., 2012, 41, 2308-2322.

2. J. Y. Lee, O. K. Farha, J. Roberts, K. A. Scheidt, S. B. T. Nguyen and J. T. Hupp, Chem. Soc. Rev., 2009, 38, 14501459.

3. M. C. Bernini, D. Fairen-Jimenez, M. Pasinetti, A. J. Ramirez-Pastor and R. Q. Snurr, J. Mater. Chem. B, 2014, 2, 766-774.

4. A. C. McKinlay, R. E. Morris, P. Horcajada, G. Ferey, R. Gref, P. Couvreur and C. Serre, Angew. Chem., Int. Ed., 2010, 49, 6260-6266.

5. H. Furukawa, K. E. Cordova, M. O'Keeffe and O. M. Yaghi, Science (Washington, DC, U. S.), 2013, 341, 974.

6. C. Wang, D. Liu and W. Lin, J. Am. Chem. Soc., 2013, 135, 13222-13234.

$7 . \quad$ L. Peng, S. Wu, X. Yang, J. Hu, X. Fu, Q. Huo and J. Guan, RSC Adv., 2016, 6, 72433-72438. 
8. S. H. Doan, K. D. Nguyen, P. T. Huynh, T. T. Nguyen and N. T. S. Phan, J. Mol. Catal. A Chem., 2016, 423, 433-440.

9. X. Li, C. Xiao, T. W. Goh and W. Huang, Abstracts of Papers, 252nd ACS National Meeting \& Exposition, Philadelphia, PA, United States, August 21-25, 2016, 2016, ENFL-485.

10 H. Ramezanalizadeh and F. Manteghi, RSC Adv., 2016, 6, 99096-99104.

11. P. Cancino, A. Vega, A. Santiago-Portillo, S. Navalon, M. Alvaro, P. Aguirre, E. Spodine and H. Garcia, Catal. Sci. Technol., 2016, 6, 3727-3736.

12. J.-C. Wang, F.-W. Ding, J.-P. Ma, Q.-K. Liu, J.-Y. Cheng and Y.-B. Dong, Inorg. Chem., 2015, 54, 10865-10872.

13. Y. Qi, Abstracts of Papers, 252nd ACS National Meeting \& Exposition, Philadelphia, PA, United States, August 21-25, 2016, 2016, ORGN-13.

14. J.-S. Wang, F.-Z. Jin, H.-C. Ma, X.-B. Li, M.-Y. Liu, J.-L. Kan, G.-J. Chen and Y.-B. Dong, Inorg. Chem., 2016, 55, 66856691.

15. P. Weerachawanasak, G. J. Hutchings, J. K. Edwards, S. A. Kondrat, P. J. Miedziak, P. Prasertham and J. Panpranot, Catal. Today, 2015, 250, 218-225.

16. L. Li, J. Zhou, Z. Xie and W. Ouyang, Shiyou Xuebao, Shiyou Jiagong, 2013, 29, 975-983.

17. H. Sakurai, K. Koga, Y. lizuka and M. Kiuchi, Appl. Catal., A, 2013, 462-463, 236-246.

18. K. M. Kadish, K. M. Smith, R. Guilard and Editors, The Porphyrin Handbook; Volume 1, Synthesis and Organic Chemistry, 2000.

19. K. M. Kadish, K. M. Smith, R. Guilard and Editors, The Porphyrin Handbook; Volume 3, Inorganic, Organometallic and Coordination Chemistry, 2000.

20. W.-Y. Gao, M. Chrzanowski and S. Ma, Chem. Soc. Rev., 2014, 43, 5841-5866.

21. B. J. Burnett, P. M. Barron and W. Choe, CrystEngComm, 2012, 14, 3839-3846.

22. O. K. Farha, A. M. Shultz, A. A. Sarjeant, S. T. Nguyen and J. T. Hupp, J. Am. Chem. Soc., 2011, 133, 5652-5655.

23. S. Nakagaki, G. K. B. Ferreira, G. M. Ucoski and K. A. D. de Freitas Castro, Molecules, 2013, 18, 7279-7308.

24. K. S. Suslick, P. Bhyrappa, J. H. Chou, M. E. Kosal, S. Nakagaki, D. W. Smithenry and S. R. Wilson, Acc. Chem. Res., 2005, 38, 283-291.

25. I. Aviv and Z. Gross, Chem. Commun. (Cambridge, U. K.), 2006, 4477-4479.

26. S. Zhu, X. Xu, J. A. Perman and X. P. Zhang, J. Am. Chem. Soc., 2010, 132, 12796-12799.

27. C.-M. Ho, J.-L. Zhang, C.-Y. Zhou, O.-Y. Chan, J. J. Yan, F.-Y. Zhang, J.-S. Huang and C.-M. Che, J. Am. Chem. Soc., 2010, 132, 1886-1894.

28. H.-Y. Thu, G. S.-M. Tong, J.-S. Huang, S. L.-F. Chan, Q.-H. Deng and C.-M. Che, Angew. Chem., Int. Ed., 2008, 47, 9747-9751.

29. H. Cui, Y. Wang, Y. Wang, Y.-Z. Fan, L. Zhang and C.-Y. Su, CrystEngComm, 2016, 18, 2203-2209.

30.

E. S. Larrea, R. Fernandez de Luis, J. Orive, M. Iglesias and M. I. Arriortua, Eur. J. Inorg. Chem., 2015, 2015, 46994707.

31.

.

M. E. Kosal, J.-H. Chou, S. R. Wilson and K. S. Suslick, Nat. Mater., 2002, 1, 118-121.

A. Fidalgo-Marijuan, E. Amayuelas, B. Bazan, M. K. Urtiaga and M. I. Arriortua, Molecules, 2015, 20, 6683-6699. W. Yinghua, J. Appl. Crystallogr., 1987, 20, 258-259. A. T. U. Ltd., Journal, 2012.

G. M. Sheldrick, Acta Crystallogr., Sect. A Found. Crystallogr., 2008, 64, 112-122.

L. J. Farrugia, J. Appl. Crystallogr., 1997, 30, 565.

A. L. Spek, Acta Crystallogr., Sect. C Struct. Chem., 2015, 71, 9-18.

S. Alvarez, D. Avnir, M. Llunell and M. Pinsky, New J. Chem., 2002, 26, 996-1009.

M. Llunel, D. Casanova, J. Cirera, J. M. Bofill, P. Alemany, S. Álvarez, M. Pinsky and D. Yatunir, Journal, 2003.

S. Alvarez, P. Alemany, D. Casanova, J. Cirera, M. Llunell and D. Avnir, Coord. Chem. Rev., 2005, 249, 1693-1708. ICCD, Journal, 2001.

M. Heidari-Golafzani, M. Rabbani, R. Rahimi and A. Azad, RSC Adv., 2015, 5, 99640-99645.

S. K. Sarkar, M. S. Jana, T. K. Mondal and C. Sinha, Appl. Organomet. Chem., 2014, 28, 641-651.

A. S. Burange, R. V. Jayaram, R. Shukla and A. K. Tyagi, Catal. Commun., 2013, 40, 27-31.

A. Wusiman and C.-D. Lu, Appl. Organomet. Chem., 2015, 29, 254-258.

R. A. Sheldon, M. Wallau, I. W. C. E. Arends and U. Schuchardt, Acc. Chem. Res., 1998, 31, 485-493. 